\title{
GMTIFS: deformable mirror environmental testing for the on-instrument wavefront sensor
}

\author{
M. Copeland ${ }^{\mathrm{a}}$, I. Price ${ }^{\mathrm{a}}$, F. Rigaut ${ }^{\mathrm{a}}$, G. Bloxham ${ }^{\mathrm{a}}$, R. Boz ${ }^{\mathrm{a}}$, D. Bundy ${ }^{\mathrm{a}}$, B. Espeland ${ }^{\mathrm{a}}$, and \\ R. Sharp ${ }^{\mathrm{a}}$ \\ ${ }^{a}$ Research School of Astronomy and Astrophysics, Australian National University, Canberra, \\ ACT 2611, Australia
}

\begin{abstract}
GMTIFS requires a deformable mirror (DM) as part of its on-instrument wavefront sensor (OIWFS). The DM facilitates wavefront correction for the off-axis natural guide star, with the objective being to maximize the energy in the diffraction core and improve the signal-to-noise ratio of the guide star position measurement. It is essential that the OIWFS be positionally stable with respect to the science field. The use of J-K to observe the guide star, and thus the need to limit thermal background, essentially requires the DM in the OIWFS to be operated at or below $-40^{\circ} \mathrm{C}$. This is below the standard operating temperature range of currently available DMs. In cooperation with the manufacturers we are testing the performance of three DMs at temperatures from ambient to $-45^{\circ} \mathrm{C}$, or cooler. In the context of the OIWFS adequate stroke, open-loop positioning stability, hysteresis, interactuator surface figure and dynamic response are key performance criteria. A test system based around high spatial sampling of the DM aperture with a Shack-Hartmann wavefront sensor has been built. The opto-mechanical design permits a DM to be contained in a cryostat so that it may be cooled in isolation. We describe this test system and the test cases that are applied to the ALPAO DM-69, Boston MicroMachines 492DM and the IrisAO PTT111 deformable mirrors. Preliminary results at ambient temperatures are presented.
\end{abstract}

Keywords: Adaptive Optics, Deformable Mirror, Shack-Hartmann, Wavefront Sensor

\section{INTRODUCTION}

GMTIFS requires a deformable mirror (DM) as part of its on-instrument wavefront sensor (OIWFS). This optic will provide a correction to high-order spatial modes for an off-axis natural guide star. The guide star position will be monitored in the $\mathrm{J}-\mathrm{K}$ passbands, so thermal emission from the optics must be limited to ensure the guide star position can be measured with at least one milli-arcsecond (mas) precision. The cryogenic and vacuum environment of the GMTIFS instrument is far from the specified operating environment of commercial-off-the-shelf (COTS) deformable mirrors, but the OIWFS must be rigidly located with respect to the science detector, and therefore it must be within the cryostat envelope. A double chambered thermal enclosure has been proposed to allow the OIWFS DM to be operated at a different temperature to the rest of the instrument, and $-40^{\circ} \mathrm{C}$ would be cool enough to limit thermal emission and satisfy the signal-to-noise requirements of the OIWFS. This is still substantially cooler than the lowest rated operating temperature of a COTS DM, but it is feasible that a COTS DM will meet the OIWFS requirements at $-40^{\circ} \mathrm{C}$.

Three different deformable mirrors will be cooled below their rated operating temperatures to assess their static and dynamic wavefront correcting performance in the context of the GMTIFS instrument. The target operating temperature is $-40^{\circ} \mathrm{C}$, but each device will be cooled from ambient temperature in steps to $-45^{\circ} \mathrm{C}$ or cooler. Performance changes will be monitored and the cooling schedule aborted if requirements are no longer being met.

Further author information: (Send correspondence to Ian Price.)

Ian Price.: E-mail: ian.price@anu.edu.au

Francois Rigaut: E-mail: francois.rigaut@anu.edu.au

Adaptive Optics Systems V, edited by Enrico Marchetti, Laird M. Close, Jean-Pierre Véran, Proc. of SPIE Vol. 9909, 990980 (c) 2016 SPIE · CCC code: 0277-786X/16/\$18 · doi: 10.1117/12.2231550 
The key performance requirements are a best-flat with wavefront error $<\lambda / 50$ at $2 \mu m$, a usable stroke of at least $2 \mu \mathrm{m}$ and an operating rate of at least $1 \mathrm{kHz}$. A test system based on a Shack-Hartmann wavefront sensor has been developed that allows a broad range of tests to be conducted without adjustment of the optomechanical setup. This has been achieved by high spatial sampling of the interactuator spacing of the DM, typically with 4-to-5 lenslets on the microlens array optically conjugated to the DM interactuator separation. This provides adequate sampling to monitor the DM surface at spatial frequencies far higher than the DM actuators can control. The other essential feature is a detector with a high frame rate with a region-of-interest at least the size of a single subaperture. This has been achieved with an sCMOS detector packaged in the form of a PCO Edge 4.2 camera. With the camera operating in rolling shutter mode frame rates above $7.3 \mathrm{kHz}$ are attained. This is sufficient to sample the dynamic response of a DM to a step change in actuator command.

The optical arrangement is similar to a Michelson interferometer, with the DM providing retro-reflection on one arm. The other arm has a reference flat for alignment purposes, but this path is blocked during test of a DM. The output arm has a simple relay that sets the spatial sampling scale and re-images the deformable mirror surface on the lenslet array. The spots formed at the focus of the lenslet array are re-imaged on the sCMOS detector by a 1:1 telecentric relay. This is required as the focal length of suitable lenslet arrays is shorter than the front-face-to-detector optical path length of the PCO Edge 4.2 device, $15.5 \mathrm{~mm}$. The input arm is a simple collimator with a $1000 \mathrm{~mm}$ focal length. A $590 \mathrm{~nm}$ LED and $200 \mu \mathrm{m}$ fibre provides an unresolved source to each subaperture. The distance between the beam splitter and the deformable mirror surface is approximately $130 \mathrm{~mm}$. This provides sufficient space to enclose just the DM in a cryogenic chamber. The chamber will be filled with $N_{2}$ at a pressure of 1 atmosphere, as some DM control systems assume there is damping from the environment. A schematic of the arrangement, excluding the relay between the lenslet array and detector and cryostat, is given in figure 1.

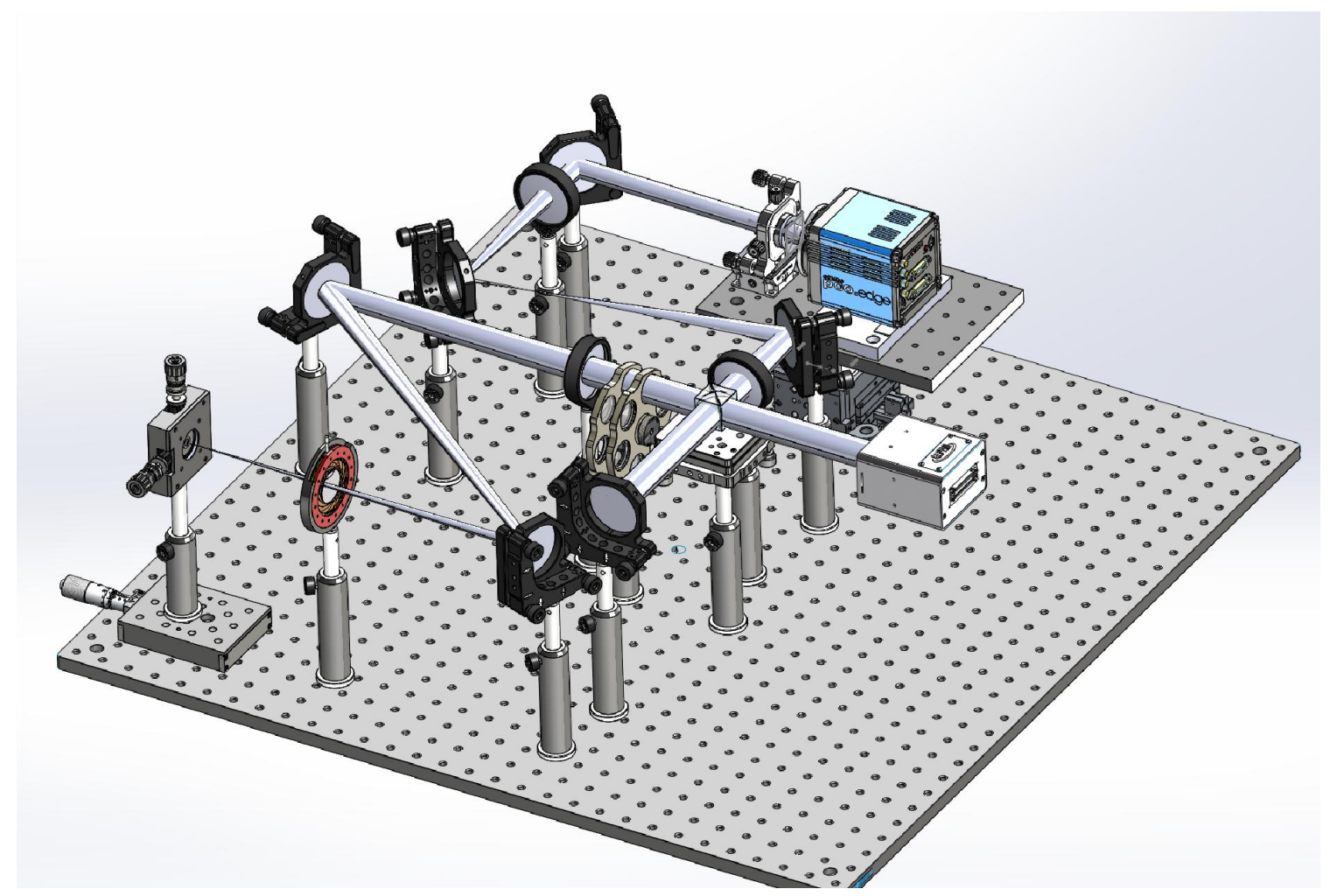

Figure 1. Solid model of the test system opto-mechanical configuration. The deformable mirror under test is positioned at the back-right on the transmissive path through the cube beam splitter. The output path includes the scale changing optical relay and Shack-Hartmann wavefront sensor. 


\section{REQUIREMENTS}

GMTIFS requires a deformable mirror within the envelope of the instrument to provide high order wavefront correction for an off-axis natural guide star. The correction imparted by this device is driven by the tomographic wavefront information from the laser tomography adaptive optics system. It accounts for the difference in wavefront aberration between the science field and the guide star, as the adaptive secondary mirror of the GMT will be correcting the wavefront to the science target. Effective guiding will only be achieved if the OIWFS is rigid with respect to the science detector. This places requirements on the mechanical rigidity of the system. It also requires the DM surface not include any tip or tilt modes. Ideally, the DM would be rigidly attached to the GMTIFS optical table, but this would place it in an extreme cryogenic environment. At a temperature of $-40^{\circ} \mathrm{C}$ the thermal emission from the DM would be low enough so as not to limit the position measurement of the guide star. While beyond the rated operating range of most COTS DMs, it is not an extreme regime, and an suitable environment can be incorporated into the overall GMTIFS design.

Although the wavefront correction required is relative to the science path, a minimum actuator stroke of $2.0 \mu \mathrm{m}$ is necessary. Although it is anticipated that the open loop response of a DM will diminish with decreasing temperature, the nature of any changes has not been systematically tested.

The GMTIFS OIWFS will require a DM with at least a 20x20 actuator array. Consultation with DM manufacturers has verified that there is no direct association between size and a capacity to operate at $-40^{\circ} \mathrm{C}$. We have therefore chosen to test smaller, thus less expensive, DMs that utilize the same actuator technology and production processes as a larger DM that meets the size requirement.

Actuator latency, or rise time, is required to be less than $750 \mu \mathrm{s}$. In this context the latency of the DM actuator is of interest, not the $\mathrm{AO}$ control system as a whole, and it is defined as the time elapsed between an actuator starting to move and reaching $90 \%$ of the steady-state displacement.

The DM must not introduce unwanted tip and tilt modes above 1mas RMS when commanding higher order modes at an update rate of $500 \mathrm{~Hz}$. The angular limit of 1 milliarcsecond is in the context of a natural guide star and the GMTIFS instrument on the GMT.

Active correction of the DM to flat must have a surface figure of $\lambda / 50$ at a wavelength of $2 \mu \mathrm{m}$.

\section{DEFORMABLE MIRRORS}

Three commercially available deformable mirrors have been identified that meet the requirements for the GMTIFS OIWFS at ambient temperature. Since the risk of permanent damage to a DM during the course of this investigation is non-trivial, expenditure on deformable mirrors has been minimized. Engineering grade devices have been purchased where a functional contiguous region of the mirror was adequate for the test purposes. The mirrors under test, and a summary of their specifications is given in table 1

Table 1. Deformable Mirror Manufacturer's Specifications

\begin{tabular}{|l|l|l|l|}
\hline & ALPAO DM-69 & BMC 492-DM & Iris AO PTT111 \\
\hline Type & Continuous & Continuous & Segmented \\
\hline Actuation Structure & Square Grid & Square Grid & Hexagonal Segments \\
\hline Aperture $(\mathrm{mm})$ & 10.5 & 9.2 & 3.5 \\
\hline Best Flat Surface $(\mathrm{nm}$ RMS $)$ & 7 & $<30$ & $<20$ \\
\hline Stroke $(\mu \mathrm{m})$ & 40 & 3.5 & 8 \\
\hline Pitch $(\mu \mathrm{m})$ & 1500 & 400 & 606 (center to center) \\
\hline Operating Temperature $(\mathrm{C})$ & -10 to 35 & - & - \\
\hline
\end{tabular}




\section{TEST CASES}

Assessment of each deformable mirror is made via a series of test cases, each of which is designed to assess a single characteristic of the mirror being tested. These are summarized in table 2. The full suite of test cases are repeated at a series of operating temperatures, starting at ambient and decreasing to $-45^{\circ} \mathrm{C}$ in $5^{\circ} \mathrm{C}$ steps. The image data is processed to slopes measurements in real-time by the control system. Sets of slope and DM command data are captured for post processing and analysis.

Table 2. Test case descriptions

\begin{tabular}{|l|l|}
\hline Test & Description \\
\hline 1 & Un-powered mirror figure \\
2 & Powered mirror figure (factory calibration flat/idle state) \\
3 & Single actuator influence function \\
4 & $3 x 3$ actuator influence function \\
5 & Best flat (correcting static aberrations in the test system) \\
6 & Interactuator stroke with applied waffle pattern \\
7 & Hysteresis \\
8 & Open loop creep \\
9 & Open loop wavefront error at $500 \mathrm{~Hz}$ \\
10 & Step response \\
\hline
\end{tabular}

The tests are described in terms of control via independent actuators, and are therefore not all applicable to the Iris AO PTT111 segmented mirror.

The test cases 1-to-9 use a configuration with a large contiguous region of the mirror being sampled by the wavefront sensor. The wavefront is reconstructed from the time-averaged measured slopes via an SVD generated reconstructor matrix at the cut-off spatial frequency of the WFS. Simple metrics, such as peak-tovalley and RMS wavefront are computed from the reconstructed wavefront, and monitored for changes with changing DM operating temperature.

Test case 10 uses a configuration with a significantly reduced set of subapertures, chosen so that the highest possible wavefront sensor frame rate can be achieved. An actuator that has an influence on the chosen subapertures is commanded with a square wave and the slopes are recorded. The slopes response from both edges over hundreds of cycles are combined. The rise time, overshot and ripple are determined from the mean step response.

The un-powered and powered mirror tests will identify any distortion of the mirror surface as a result of thermal contraction of the mirror structure. Any distortion would ultimately be part of the static aberration corrected by the DM. Since this reduces the stoke available for dynamic correction it is significant for GMTIFS.

The single actuator and $3 \times 3$ block influence function tests will identify any change in the open-loop spatial response with temperature.

The best flat test will determine if cooling results in any uncorrectable high order modes in the surface figure. For this test the system operates in closed loop.

The waffle pattern induces the greatest stress on the mirror and is the test mode most likely to result in failure of the DM. While it is not the intention to test mirrors to the point of failure, this test may help identify failure modes.

Since the GMTIFS OIWFS will operate open-loop, an accurate actuator hysteresis model is essential. The hysteresis test will ascertain if this model has a temperature dependence.

The open-loop creep test will apply a constant non-zero command to the DM and monitor the slopes for 120 minutes. This will identify any drift in the surface figure with time.

The open-loop and step response tests will determine if the mirror actuator rise time changes with temperature, and whether the DM can provide active correction at $500 \mathrm{~Hz}$. 


\section{OPTICS, CONTROL AND DATA ANALYSIS}

The traditional approach to measuring the surface figure of deformable mirrors is with an interferometer, but for this experiment a Shack-Hartmann wavefront sensor have several advantages. The response is linear; the dynamic range is a function of the optical design; the reconstruction process is well understood and is straight forward to calibrate; a control system with most of the required functionality was in-hand. While it is possible that cooling a DM will affect the mirror figure, it is not expected to result in significant structure at very high spatial frequencies, largely due to the elastic nature of the mirror substrate. In the case of segmented mirrors some bending of the segment may occur and the Shack-Hartmann wavefront sensor is sensitive to these low order modes.

Though not formally required, it is practical to align the lenslet array grid to the pixel grid of the detector and define the subaperture boundaries along pixel boundaries. The lenslet array is mounted in a translation stage mount on a goniometer. The camera is mounted on an $\mathrm{X}-\mathrm{Y}-\mathrm{Z}$ stage. Together this allows alignment of the subaperture grid to the DM aperture and the detector to the lenslet array grid. The lenslet array pitch of $150 \mu \mathrm{m}$ is not an integer multiple of the pixel size $(6.5 \mu \mathrm{m})$, but is close, so a $4 f$ relay between lenslet array and detector was chosen. By introducing a small amount of defocus in the wavefront at the lenslet array the spot images can be scaled and the subaperture size set at 23 pixels. The defocus is introduced by adjusting the separation between the relay lenses between the DM and the lenslet array. The diffraction limited PSF of each spot from the lenslet array has a FWHM of more than two pixels so the spot position can be measured without quantization error. The optical system is semi-enclosed on the sides and completely shielded above and below. This serves both as baffling against stray light and airflow from the various device cooling systems and the lab environment. The baffling has reduced the contribution from turbulence to the wavefront to $<50 \mathrm{~nm}$ RMS.

Supporting both the step response and best-flat test cases requires a control system with a configurable system geometry and the capacity for closed loop control. A control system architecture based on an Intel PC with expansion cards to support both the wavefront sensor and deformable mirror interfaces has been utilized at RSAA in several adaptive optics instruments. ${ }^{1}$ This control system architecture was adapted for the test system. Data acquisition, processing, control and telemetry are cooperative asynchronous tasks within a single process space. Both control and telemetry use a client-server model, with the ZeroMQ middleware used to facilitate in both cases. This allows human interaction via GUI tools, but it is also the mechanism used to run the test cases and monitor progress. The telemetry streams provide regular snapshots of the state of the system at approximately $30 \mathrm{~Hz}$, but complete records of the core control system cycle are captured by the control system and are written to file on demand. This record has data at the image capture frame rate and we refer to these as data sequences. They are the primary source of data for analysis.

The most significant conceptual change from the original code-base was supporting run-time reconfiguration of the system geometry. Though simple, this had the side effect that algorithms carefully optimized for a specific project geometry and closed loop frame rates in excess of $2 \mathrm{kHz}$ could not be utilized. The additional time spent processing data increased the control system latency, but the sCMOS camera readout is still the frame rate limiting factor. Since only static aberrations are corrected in a closed-loop operating mode the increased latency is inconsequential.

The most important feature of the control system is the ability to inject disturbances into the deformable mirror command stream. Input files representing time series of actuator command disturbances are generated off-line and can be loaded on demand. Influence function, step response, hysteresis, creep and interactuator stroke test cases are all based on specific disturbance time series.

Each test case is executed from a host, remote from the control system hosts, using a dedicated data acquisition sequence. These acquisition sequences are directed using a combination of control system commands and ssh/scp system calls. The data acquisition sequences generate data files that are copied and used as input to the data reduction process. All the acquisition sequences, written in yorick and using the yao libraries, ${ }^{2}$ are automated and can be tailored to the specific needs. The typical flow of an individual test case is a configuration step, acquisition, data transfer, data reduction and generation of figures and summary results. Most data acquisition sequence have a duration of approximately one minute, with the exception of the open loop creep test case which takes up to three hours. At the completion of the set of test cases the results from individual test are collated in a web page and published for internal use. 
An important and unavoidable data reduction step is the phase reconstruction from the high order ShackHartmann wavefront sensor. We used $\mathrm{yao}^{2}$ to generate a reconstructor with a virtual set of actuators in the Fried geometry configuration applicable to our Shack-Hartmann wavefront sensor. We used a SVD reconstructor, compatible with the kind of open-loop, one step reconstruction that is necessary for this problem. We found that partially illuminated edge subapertures introduced a reconstruction bias that limited the phase reconstruction accuracy unless the partial illumination was well modelled. These biases are usually in the form of high frequency ripples being propagated inside the pupil from edge reconstruction errors. Since any change in the optical alignment between the DM and WFS changes the illumination of edge subapertures, we chose to remove edge subapertures from the reconstruction, rather than attempt to accurately model them. In the case of the ALPAO DM- 69 , this reduces the subaperture grid from $36 \times 34$ to $34 \times 34$ and the phase reconstructions has an accuracy of a few nanometers.

Our goal in the design and realization of the data acquisition and reduction tooling was to make is as DM agnostic as possible. This goal has been largely fulfilled, in the sense that the data acquisition and reduction software queries the control system for the subaperture configuration, and is independent of the actual DM.

\section{PRELIMINARY RESULTS}

The test system has been configured for the ALPAO DM-69 and the test cases have been extensively executed as part of the development and refinement of the automated testing regime. The results obtained form a reference for comparison with future measurements. A subset of the results from analysis of data collected with the system are given in Figures 2 and 3
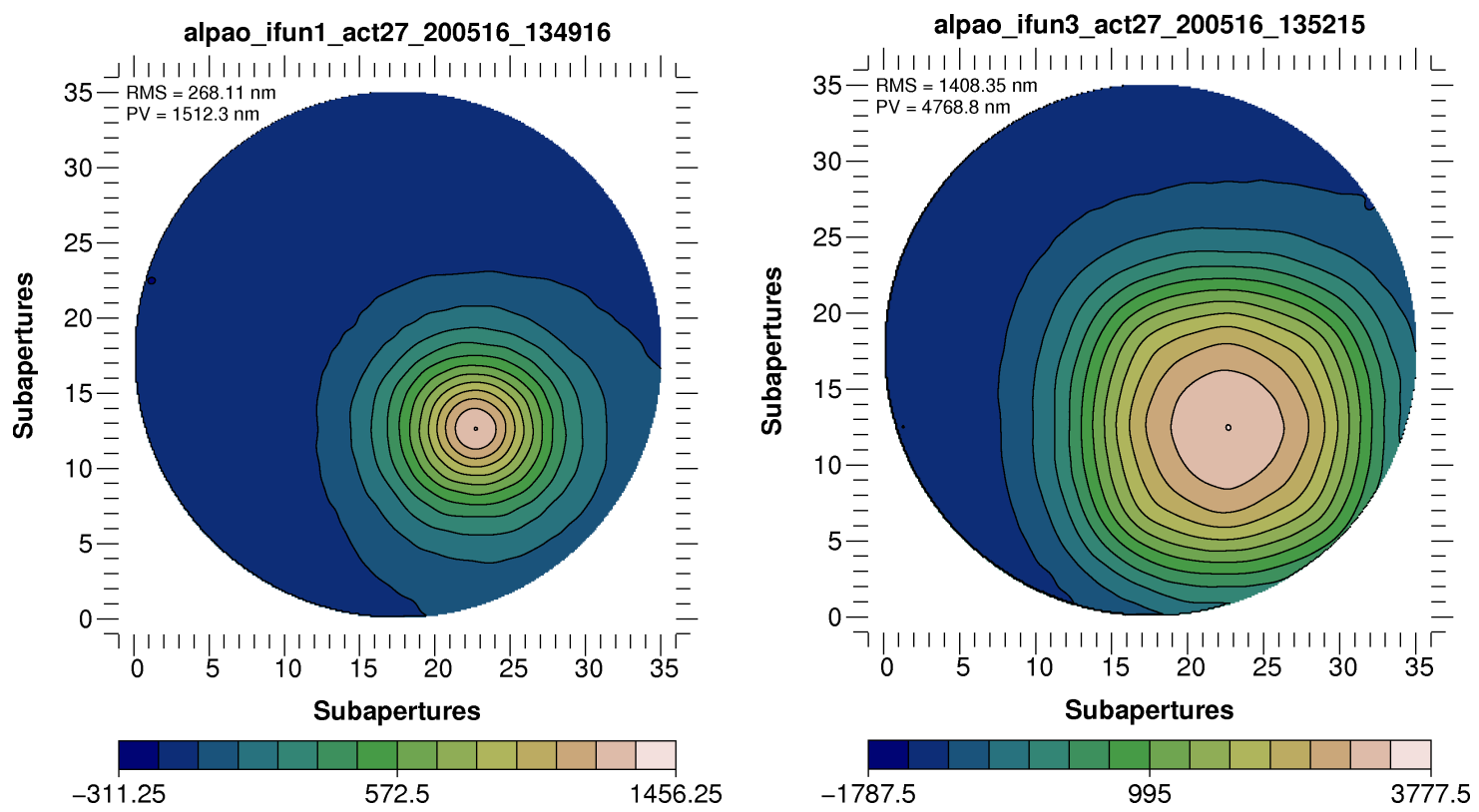

Figure 2. Indicative wavefront reconstruction results from tests of the ALPAO DM-69 at ambient temperature. Single actuator (left) and $3 \times 3$ block actuation (right) influence functions.

\section{CONCLUSIONS}

Results from tests at ambient temperatures have demonstrated that a single test system will allow semiautomated and systematic measurement of a broad range of performance characteristics of a deformable mirror as it is cooled to $-40^{\circ} \mathrm{C}$. The use of Shack-Hartmann sensor technology with high spatial sampling compared to the Fried geometry leverages both a control system developed for real-time AO correction, and traditional AO data analysis methodologies. 

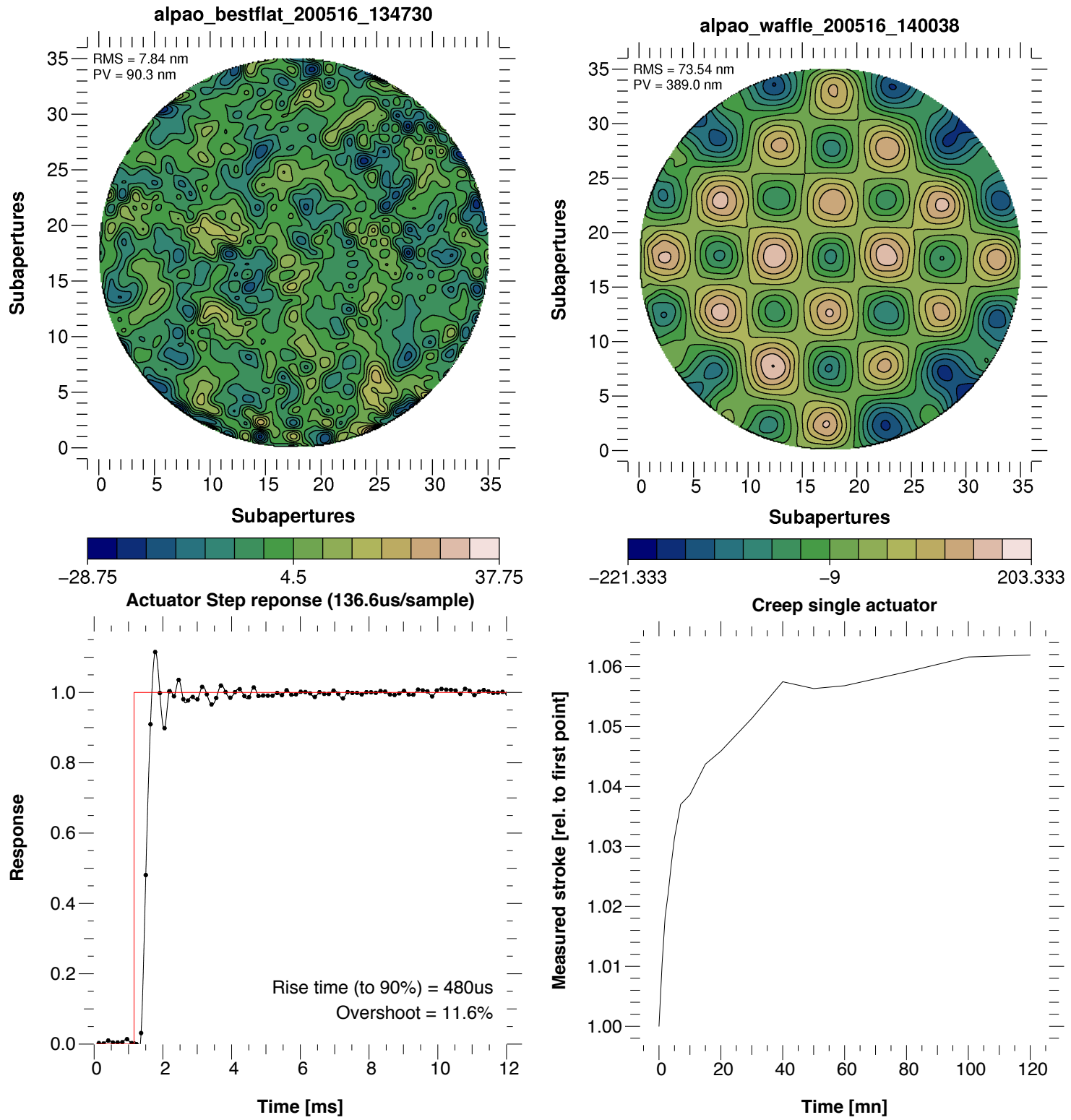

Figure 3. Indicative results from tests of the ALPAO DM-69 at ambient temperature. Best flat wavefront (top left), waffle pattern wavefront (top right), steper response (bottom left) and single actuator creep (bottom right).

\section{ACKNOWLEDGMENTS}

This work is funded by the Giant Magellen Telescope Organisation and we thank them for their ongoing support. We also thank ALPAO, Boston Micromachines Corporation and Iris AO for their assistance and support of this work.

\section{REFERENCES}

[1] Bennet, F., D'Orgeville, C., Gao, Y., et al., "Adaptive optics for space debris tracking," in [Adaptive Optics Systems IV], Marchetti, E., Close, L., and Veran, J., eds., Proc. SPIE 9148 1F, 1-9 (2014).

[2] Rigaut, F. and van Dam, M., "Simulating astronomical adaptive optics systems using yao," in [3rd Conference on Adaptive Optics for Extremely Large Telescopes], AO4ELT (2013). 